\section{Multilingual website and cyberconsultations for oromandibular dystonia}

\author{
Kazuya Yoshida \\ Department of Oral and Maxillofacial \\ Surgery, National Hospital \\ Organization, Kyoto Medical Center, \\ Japan
}

\begin{abstract}
Oromandibular dystonia is a focal dystonia that manifests as involuntary masticatory and/or tongue muscle contractions. This movement disorder is frequently misdiagnosed as a temporomandibular disorder. Hence, it would be useful to establish a method that makes it possible for patients with the condition to find appropriate medical institutions by themselves. The author produced a website Involuntary movements of the stomatognathic region (https://sites. google.com/site/oromandibulardystoniaenglish/) for patients with oromandibular dystonia, which is available in twenty languages. It has been viewed more than $1,000,000$ times by individuals from all over the world. The visitors to the site have completed questionnaires and/or sent images or videos of their involuntary movements over the internet. Cyberconsultations (remote diagnosis) were also performed via Skype $^{\mathrm{TM}}$. Approximately 1000 patients with involuntary stomatognathic movements visited our department. Only $12.5 \%$ of the patients had previously been diagnosed with or were suspected to have dystonia. The findings of this study suggest that the multilingual website has contributed to increasing awareness of oromandibular dystonia and that the provision of basic telemedicine via the internet can aid the diagnosis and treatment of oromandibular dystonia.
\end{abstract}

\section{Introduction}

Dystonia is a movement disorder characterized by sustained or intermittent muscle contractions that cause abnormal, often repetitive, movements, postures, or both. ${ }^{1}$ Dystonia is classified into various types based on two main factors: 1) its clinical characteristics, including its age at onset, bodily distribution, temporal pattern, and associated features, and 2) its etiology, e.g., whether it was caused by a pathological neurological condition or was inherited. 1
Oromandibular dystonia is a focal dystonia involving the masticatory and/or lingual muscles. . $^{2}$ It clinically presents as jaw closing dystonia, jaw opening dystonia, jaw deviation dystonia, jaw protrusion dystonia, lingual dystonia, or a combination of these abnormal movements. ${ }^{2-7}$ It interferes with chewing, swallowing, and speaking, resulting in social embarrassment and cosmetic disfigurement. Oromandibular dystonia is predominantly found in females-7 and typically occurs between the ages of 45 and 70 years. ${ }^{4}$ It has previously been treated with various antispasmodic or anticholinergic agents, but the responses to these treatments were unsatisfactory. ${ }^{3}$ The injection of botulinum toxin is the standard therapy for focal dystonias, such as blepharospasm, cervical dystonia, and oromandibular dystonia. $2,4,6-8$ Muscle afferent block therapy (the injection of a local anesthetic) has also been demonstrated to be effective against oromandibular dystonia and can be used as a predictor of the response to botulinum therapy.3,5 Surgical interventions (coronoidotomy) have proven to be very effective against severe jaw closing dystonia combined with trismus. 9,10

Unfortunately, oromandibular dystonia is often misdiagnosed as temporomandibular disorder, psychogenic disease, bruxism, or temporomandibular joint ankyloses.3,5 Therefore, patients with the condition often visit many hospitals and departments over long periods before being correctly diagnosed. Consequently, they do not receive appropriate treatment, and their conditions deteriorate.3,5,10 In addition, most of the attending physicians in such cases do not realize that they have misdiagnosed their patients. First of all, it is necessary for both patients and physicians to recognize the existence of oromandibular dystonia. In addition, we must establish a method that enables patients with the condition to find appropriate medical institutions by themselves, even if they do not live near them. The growing disparity between the demand for adequate treatment for oromandibular dystonia and the lack of specialists means that innovative strategies are required to deliver appropriate medical care for the condition. The American Telemedicine Association formally defines the term telemedicine as the exchange of medical information from one site to another via electronic communications to improve a patient's clinical health status. ${ }^{11}$ As a result of advances in information technology, it has become very easy to communicate across the internet using software such as $\mathrm{Skype}^{\mathrm{TM}}$. Recently, medical tourism; i.e., when patients visit different regions or countries to obtain medical services, has become increasingly popular.
Correspondence: Kazuya Yoshida, Department of Oral and Maxillofacial Surgery, National Hospital Organization, Kyoto Medical Center, 1-1 Mukaihata-cho, Fukakusa, Fushimi-ku, Kyoto 612-8555, Japan.

Tel.: +81.75.6419161 - Fax: +81.75.6434325.

E-mail: kayoshid@kyotolan.hosp.go.jp

Key words: oromandibular dystonia, internet, telemedicine, remote diagnosis, medical tourism.

Conflict of interest: the author declares no conflict of interest.

Funding: This study was supported by grants from the Japanese Ministry of Health, Labor, and Welfare (15K09370, 24592946 and 22111201)

Received for publication: 14 December 2017. Accepted for publication: 25 December 2017.

This work is licensed under a Creative Commons Attribution NonCommercial 4.0 License (CC BY-NC 4.0).

CCopyright K. Yoshida., 2018

Licensee PAGEPress, Italy

Neurology International 2018; 10:7536

doi:10.4081/ni.2018.7536

Medical tourists seek a variety of medical treatments abroad, particularly cosmetic surgery or dental treatment. ${ }^{12}$ Therefore, visiting appropriate hospitals abroad might be an option for patients that require treatment for involuntary contractions of the stomatognathic system. In this report, the author describes the remote diagnosis of oromandibular dystonia via the internet.

\section{Materials and Methods}

\section{Website about oromandibular dystonia}

In November 2011, the author used Google Sites to produce a website [Involuntary movements of the stomatognathic region (https://sites.google.com/site/ oromandibulardystoniaenglish/)] for patients with oromandibular dystonia and other movement disorders affecting the orofacial region. Japanese, German, and French versions of the website were also produced in the same fashion. The author gradually produced versions in other languages. Currently, the website is available in twenty languages: English, Japanese, German, French, Spanish, Russian, Portuguese, Italian, Arabic, Chinese, Korean, Hindi, Thai, Dutch, Swedish, Danish, Turkish, Vietnamese, Polish, and 
Czech (Supplementary Table S1). On the site, the author explains about conditions that cause involuntary movements of the stomatognathic system, such as oromandibular dystonia and dyskinesia, and their symptoms, diagnosis, and treatment. In addition, the site provides information about hospitals that are capable of treating dystonia, access to our clinic, medical tourism, and online educational resources for patients. Each version of the site contains 8 YouTube videos of typical involuntary movements before and after treatment, which the visitors can easily access.

\section{Telemedicine}

Oromandibular dystonia is clinically diagnosed based on its characteristic features of focal dystonia, a stereotyped pattern of muscle contraction, task-specificity, sensory ticks, the overflow phenomenon, morning benefit, and co-contraction. ${ }^{3-5}$ Questions about these clinical features were included in a 16-question questionnaire about oromandibular dystonia. The questionnaire also included questions concerning the user's symptoms and past examination history, treatment, other movement disorders, and psychogenic disease. If a visitor to the site wanted to be diagnosed by the author, they were asked to fill out the questionnaire and email, fax, or post it to the author. In addition, they were able to make a video or take photos of their involuntary movements and email them as attachments (Figure 1).

\section{Remote diagnosis}

Patients can also communicate with us via the free voice over internet protocol software program Skype ${ }^{\mathrm{TM}}$, which is an application that is mainly used to provide video chat and voice call services. It also has an instant messaging function, which makes it possible to exchange text or video messages, files, and images. Cyberconsultations or remote diagnosis can also be attempted via the internet using Skype $^{\mathrm{TM}}$ (Figure 1). The author performs remote diagnostic assessments after providing a full explanation about the data confidentiality risks associated with using a public system for patient-physician interactions. Cyberconsultations are available after hospital hours (after 18:00 Japanese time). If patients have the typical symptoms of dystonia, the author will be able to determine whether they are likely to have dystonia based on the information, images, questionnaire responses, and videos they provide. Such cyberconsultations can also be used to rule out other diseases such as psychogenic diseases, temporomandibular disorders, trauma or inflammation in the orofa- cial region, and other neurological disorders. If the author suspects such conditions, the author can advise the patient to consult a specialist in a hospital near to where they live. Furthermore, the author can recommend patients with generalized dystonia or whose symptoms are not indicated for therapy involving our stomatognathic system (i.e., those that are indicated for deep brain stimulation or another neurosurgical procedure) to visit a neurosurgeon to undergo surgical treatment. The author has also added the following note about the limits of remote diagnosis to the site: Please note that we cannot be held responsible for remote diagnoses based solely on questionnaire results and/or images.

The author takes care to manage patients' personal information in an appropriate manner. Any images or video data received are stored on a password-protected computer in a responsible manner. The author keeps the computer room locked when unattended and always strives to prevent theft.

\section{Consultation}

To obtain a definitive diagnosis, the patients must visit our department to undergo electromyography; muscle palpation; and other tests such as X-rays, computed tomography scans, and magnetic resonance imaging scans, if necessary (Figure 1). Furthermore, in some cases consultations with the departments of Neurology, Psychiatry, or Neurosurgery are also required.

Botulinum toxin therapy is possible as an outpatient. Surgical procedures, such as coronoidotomy for severe trismus, require a two-week stay in hospital for the purpose of relieving limited mouth opening. 9,10

\section{Results}

The numbers of visitors to each language version of our website are shown in Figure 2. The Japanese version was the most frequently visited version (accounting for $23.8 \%$ of visitors), followed by the English version (17.6\%), the Arabic version $(8.8 \%)$, the Italian version $(7.9 \%)$, the French version $(6.8 \%)$, and the Russian version $(3.3 \%)$ (Figure 2). Figure 3 shows the numbers of visitors to the present website from each country. Japanese visitors accounted for $24 \%$ of the people that visited the website, followed by those from the USA (17.5\%), Italy (9.2\%), France (5.4\%), Russia (3.6\%), Saudi Arabia (2.8\%), and China (2\%) (Figure 3). However, $12.9 \%$ of the patients did not choose a country set- ting. The estimated total number of views of all versions of the site was $>1,000,000$, and the visitors came from over 190 countries and regions around the world (Figure 3). The YouTube videos (in English or Japanese) embedded in the site were viewed approximately 300,000 times. The author received 5,303 emails, 314 letters, and 132 faxes from visitors to the site. Only 4 emails were sent from medical professionals (a physician, an oral and maxillofacial surgeon, a dentist, and an occupational therapist). The other emails were from patients or their family members. About 180 cyberconsultations were performed using Skype $^{\mathrm{TM}}$, and a total of 1,020 patients with involuntary movements of the orofacial region visited our department. Of these, 72 patients were hospitalized, and 138 patients stayed at hotels near the hospital and visit our department as outpatients.

The rate of a previous diagnosis of the patients with oromandibular dystonia is displayed in Figure 4. The patients had consulted a mean of 3.9 departments or hospitals (range: 2-30). The consulted departments included the departments of dentistry $(69.1 \%)$, oral and maxillofacial surgery $(59.4 \%)$, neurology $(59.0 \%)$, psychiatry $(32.2 \%)$, neurosurgery $(24.9 \%)$, otorhinolaryngology (18.5\%), acupuncture (18.3\%), internal medicine $(7.0 \%)$, orthopedics $(3.7 \%)$, pain clinics $(2.8 \%)$, ophthalmology $(2.3 \%)$, rehabilitation $(1.9 \%)$, plastic surgery $(1.2 \%)$, anesthesiology $(0.9 \%)$, and others $(2.3 \%)$.

The author was able to effectively diagnose and treat the patients because we were able to exclude other diseases beforehand based on the images and video recordings of the patients' involuntary movements and remote diagnosis via the internet. The patients' diseases included temporomandibular disorders, psychogenic disease, inflammation, trauma, and sleep disorders. The author recommended the patients that were suspected to have other neurological diseases to consult neurologists near to where they lived. As a result, some patients were diagnosed with neurological diseases, including Parkinson's disease, myasthenia gravis, epilepsy, and Guillain-Barre syndrome, by neurologists. The author applied a comprehensive range of treatments including medication, botulinum therapy $\left(\right.$ Botox $\left.^{\circledR}\right), 4,8,13-15$ muscle afferent block therapy, 3,5 splint therapy 16 and oral and maxillofacial surgery. 9,10

\section{Discussion}

The present study is the first regarding a website for patients with involuntary move- 
ment of the stomatognathic system and the remote diagnosis of oromandibular dystonia via voice over internet protocol services such as Skype ${ }^{\mathrm{TM}}$.

Oromandibular dystonia is often misdiagnosed as temporomandibular disorder or psychogenic disease. 3,5 The precise etiology of oromandibular dystonia remains unclear. ${ }^{17-19}$ Most of the attending healthcare professionals in such cases do not realize that they have misdiagnosed their patients. Despite numerous studies on the epidemiology of dystonia, accurately determining the prevalence of the condition for health service planning purposes remains a significant challenge. ${ }^{20}$ Current estimates of the prevalence of oromandibular dystonia are very likely to be underestimates of the true prevalence of the condition. Only a limited number of physicians specialize in involuntary movements, even among neurologists. Thus, few neurologists are able to diagnose and treat dystonia. Even when neurologists are able to diagnose oromandibular dystonia, it is difficult to diagnose cases in which the muscles of the jaw and/or mouth exhibit abnormal contractions, and it requires skill to accurately inject botulinum toxin into the affected muscles. Therefore, very few patients with this type of dystonia are adequately diagnosed and treated. Even if patients are diagnosed correctly, they are usually treated by neurologists rather than specialists. Thus, oromandibular dystonia exists in a blind spot between medicine and dental medicine. As patients with oromandibular dystonia often first present to dental clinicians, the author attempted to improve the awareness of this condition among dental specialists, but neither dentists nor oral and maxillofacial surgeons exhibited any interest in oromandibular dystonia. Consequently, the author decided to establish a method that would allow patients to find appropriate medical institutions by themselves, even when they live far away. It is important to raise awareness of this type of dystonia among both patients and healthcare professionals using social networking. Therefore, the author produced a multilingual website about the symptoms, diagnosis, and treatment of oromandibular dystonia in order to provide a tool that could be accessed by a large percentage of the people that are suffering from or interested

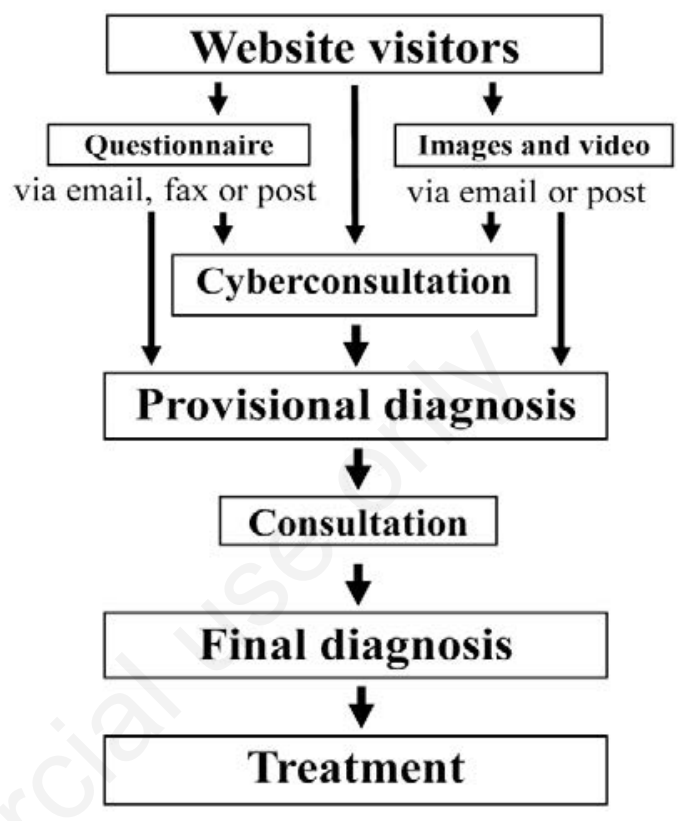

Figure 1. Procedure from diagnosis to treatment. We exclude diseases such as temporomandibular joint disorder or psychogenic disease based on information such as the patient's questionnaire responses, images, and video, and then conduct further cyberconsultations via Skype ${ }^{\mathrm{TM}}$. A final diagnosis is made after the patient visits our clinic.

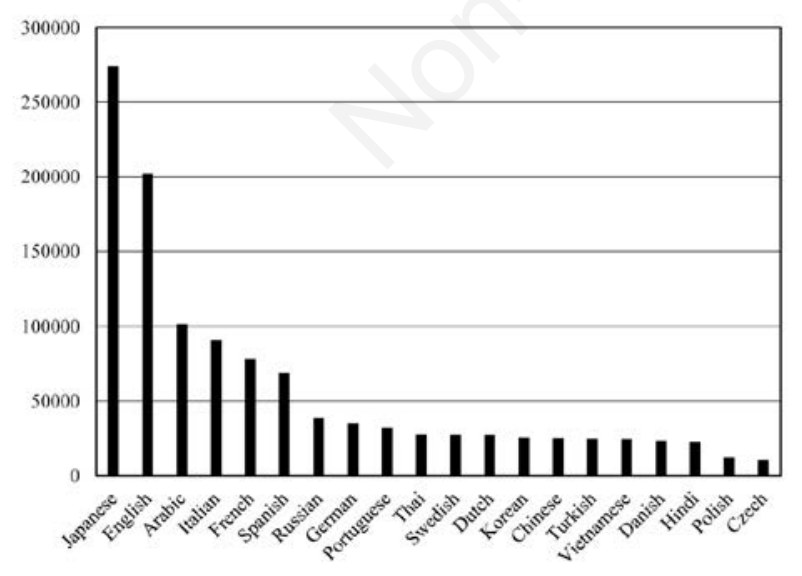

Figure 2. Number of visitors to each language version. The Japanese version of the site was the most popular, accounting for $\mathbf{2 3 . 8 \%}$ of visitors, followed by the English version (17.6\%), the Arabic version $(8.8 \%)$, the Italian version $(7.9 \%)$, the French version $(6.8 \%)$, and the Russian version $(3.3 \%)$.

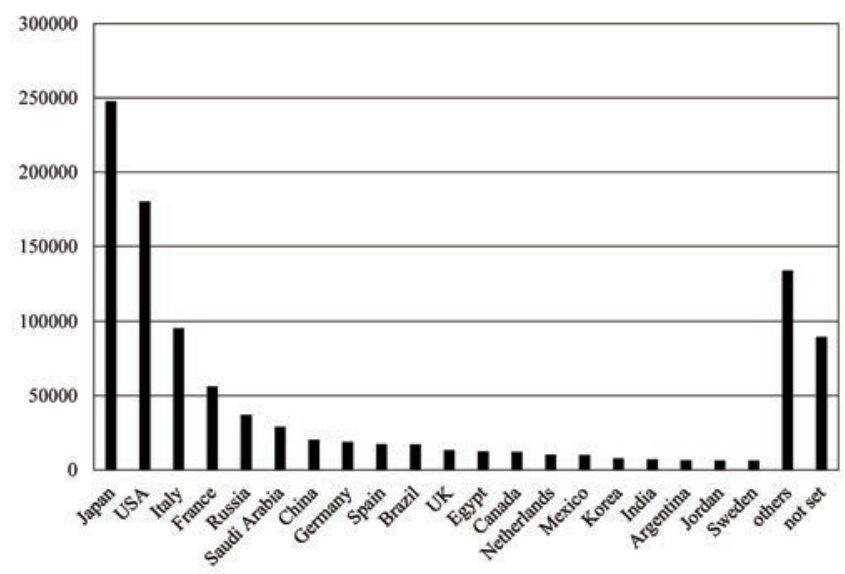

Figure 3. Number of visitors from each country. The top 20 countries in terms of the number of visitors to our site are shown. Japanese visitors accounted for $24 \%$ of the visitors to our site, followed by visitors from the USA $(17.5 \%)$, Italy $(9.2 \%)$, France (5.4\%), Russia (3.6\%), and Saudi Arabia (2.8\%). However, $12.9 \%$ of the site's visitors did not indicate their country of residence. 
in the condition. The response to this site was far beyond the author's expectations. Thus, there must be many patients with oromandibular dystonia around the world who have not been diagnosed or adequately treated. Such patients are desperate for information on oromandibular dystonia. On the other hand, only 4 of the over 5000 pieces of correspondence that the author received were from medical professionals (a physician, an oral and maxillofacial surgeon, a dentist, and an occupational therapist). They asked the author how they should diagnose and treat their patients. The author thought that many more healthcare providers would contact the author. Only $12.5 \%$ of the patients had been diagnosed with or were suspected to have dystonia before they visited our clinic (Figure 4). Unfortunately, the awareness of this condition among physicians remains surprisingly low. Physicians should get information from evidenced-based literature and other well established and/or trusted resources rather than from the internet. Evidencebased data about oromandibular dystonia is very scarce compared with the amount of information available about other types of focal dystonia such as blepharospasm or cervical dystonia. Therefore, it is important to treat more patients with oromandibular dystonia and perform clinical studies with a high level of evidence. Such studies might result in an increase in physicians' interest in the condition.

When internet use first started to become more common among the public, the vast majority of users communicated in English. However, the frequency of the use of English on the internet has gradually decreased, as the number of users in developing countries has increased rapidly. There have been particularly marked increases in the use of Arabic, Spanish, and Chinese. Indeed, only $17.6 \%$ of the visitors to the present website visited the English version (Figure 3 ). Thus, more than $80 \%$ of the visitors accessed sites in other languages. Although specialists and healthcare professionals can often communicate in English, e.g., to publish new scientific findings or share clinical information, patients around the world often communicate in other languages. Thus, it would be best if they were able to access information and resources in their mother tongues. Therefore, the present site is currently available in twenty languages (the twenty most frequently used languages on the internet). As a result, approximately $90 \%$ of internet users; i.e., 3 billion people, can access our site. The present site has been accessed by many viewers from all over the world (Figure 3). Thus, although the site is very simple and was produced using Google Sites, which is free and does not require the skills of a professional web designer, it has helped considerably to increase awareness of oromandibular dystonia among international patients. Many patients with this type of dystonia are eager to obtain information about the condition. The present site received higher numbers of visitors from Japan and Italy than the author would have expected based on the sizes of their populations (Figure 3). This might have been due to the fact that the site was linked to patient groups in these countries and that these groups recommended that patients visit the site. It might be necessary to ask neurological and movement disorderrelated patient groups and scientific societies to add a link to the present site to their homepages.

Telemedicine for Parkinson's disease and many other diseases has become prevalent in developed countries.11,21,22 Historically, telemedicine relied on expensive equipment to connect patients to a specialist in pilot programs in wealthy nations. ${ }^{21}$ The more simple the technique, the more people can access telemedicine. Therefore, it would be best if patients could communicate via the internet using free software. Telemedicine offers patients a convenient service, a reduced hospital stay, fewer office visits, and a minimal travel burden. ${ }^{21}$ Focal dystonia is generally diagnosed based on its clinical features such as a stereotyped pattern of muscle contraction, task-specificity, sensory ticks, morning benefit, etc. The first limitation of the approach that needs to be clearly understood is that it is not possible to practice medi-

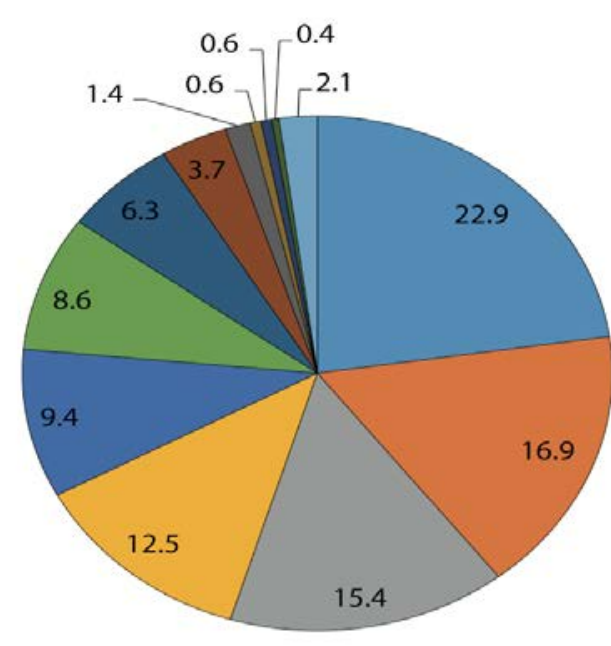

- temporomandibular disorders unknown etiology bruxism $\square$ dystonia dyskinesia psychogenic disorders occlusal problem normal - pericoronitis trigeminal neuralgia

chronic pain glossodynia other conditions

Figure 4. Percentage of alternative or uncertain diagnoses before the patients visited our clinic. Only $12.5 \%$ of the patients had been diagnosed with or were suspected to have dystonia before they visited our clinic. The most frequent misdiagnosis was temporomandibular disorders (22.9\%). 
tion about rare diseases and in aiding communication between patients and healthcare providers. Cyberconsultations with patients can provide experts with an expanded pool of patients from which to recruit for research and treatment studies. ${ }^{22}$ Such consultations also widen one's professional network; i.e., the referring physicians are likely to send additional patients and provide a larger platform for research. 22 The collection of patients with oromandibular dystonia via telemedicine and medical tourism could be very important. At our department, the author specializes in involuntary movements of the orofacial area. The author administers a comprehensive range of treatments for such involuntary movements, including medication, muscle afferent block therapy, $3,5,19$ botulinum toxin therapy, 8,15 splint therapy 16 and surgery. 9,10 There are no hospitals or departments that specialize in oromandibular dystonia. Accordingly, many patients with involuntary movements of the orofacial region are referred to the author from all over Japan and indeed the world. However, it would be impractical for patients to repeatedly visit our institution for multiple botulinum toxin injections. The author applies a precise and easy to perform method for injecting botulinum toxin involving the use of a computer-aided design/computer-aided manufacturingderived needle guide, which have been used as surgical templates for dental implant placement. 8 If local physicians could perform such injections using a needle insertion guide, it would be possible to reduce the economic burden and time constraints associated with repeated long-distance health tourism. Such needle guides can be used repeatedly (in the same patient) after re-sterilization. In cases in which a physician is able to send data about the patient's computed tomography findings and a plaster model of their maxilla, the author could prepare an insertion guide at the patient's first visit. 24 In the near future, technical developments will make it possible to directly import digital surface data generated by intra-oral scans. As a future strategy, the author is aiming to perform cyberconsultations with patients all over the world, which will be conducted after telediagnosis based on the provision of the necessary data via the internet. Such patients could then visit our clinic as medical tourists and undergo botulinum toxin therapy with the needle guide. The follow-up examinations and subsequent injections could be conducted by the patients' attending physicians.

There are various issues associated with telediagnosis and medical tourism. First, issues of distant treatment legality and license necessity in some countries. Second, adverse events can occur during medical treatment abroad, which raises medicolegal and insurance issues, as well as concerns regarding patient follow-up. Third, the impact of medical tourism has raised ethical issues, particularly regarding its impact on local health services. ${ }^{12}$ In addition, patients from abroad are not covered by the Japanese health insurance system, and only a few patients can afford to pay their own medical costs. Thus, there is a need to amend the Medical Practitioners Act. Forth, transmitting personal information over the internet carries a risk of the information being lost or disclosed to third parties, although any images and video data received are stored on a password-protected computer in a responsible manner. Furthermore, the author keeps the computer room locked when unattended and always strives to prevent theft. Finally, the author provides the remote diagnosis service on a volunteer basis. Obviously, if this type of telemedicine were applied to other fields of clinical medicine, then adequate reimbursement for the procedure would be required. To overcome the abovementioned issues, further experience and an evidence-based study involving sufficient data are required.

\section{Conclusions}

It was suggested that internet-based telemedicine is useful for the diagnosis and treatment of oromandibular dystonia.

\section{References}

1. Albanese A, Bhatia K, Bressman SB, et al. Phenomenology and classification of dystonia: a consensus up date. Mov Disord 2013;28:863-73.

2. Blitzer A, Brin M, Greene PE, Fahn S. Botulinum toxin injection for the treatment of oromandibular dystonia. Ann Otol Rhinol Laryngol 1989;98:93-7.

3. Yoshida K, Kaji R, Kubori T, et al. Muscle afferent block for the treatment of oromandibular dystonia. Mov Disord 1998;13:699-705.

4. Tan EK, Jankovic J. Botulinum toxin A in patients with oromandibular dystonia Long-term follow-up. Neurology 1999;53:2102-7.

5. Yoshida K, Kaji R, Shibasaki H, Iizuka T. Factors influencing the therapeutic effect of muscle afferent block for oromandibular dystonia and dyskinesia: implications for their distinct pathophysiology. Int J Oral Maxillofac Surg 2002;31:499-505.
6. Sinclair CF, Gurey LE, Blitzer A. Oromandibular dystonia: long-term management with botulinum toxin. Laryngoscope 2013;123:3078-83.

7. Yoshida K. Clinical and phenomenological characteristics of patients with taskspecific lingual dystonia: possible association with occupation. Front Neurol 2017;8:649

8. Yoshida K. How do I inject botulinum toxin into the lateral and medial pterygoid muscles? Mov Disord Clin Pract 2017;4:285.

9. Yoshida K. Coronoidotomy as treatment for trismus due to jaw-closing oromandibular dystonia. Mov Disord 2006;21:1028-31.

10. Yoshida K. Surgical intervention for oromandibular dystonia-related limited mouth opening: long-term follow-up. J Craniomaxillofac Surg 2017;45:56-62.

11. Mutgi SA, Zha AM, Behrouz R. Emerging subspecialties in neurology: telestroke and tele neurology. Neurology 2015;84:191-3.

12. Leggat P. Medical tourism. Aust Fam Physician 2015;44:16-21.

13. Jankovic J, Brin MF. Therapeutic uses of botulinum toxin. $\mathrm{N}$ Engl $\mathrm{J}$ Med 1991;324:1186-94.

14. Jankovic J, Schwartz K, Donovan DT. Botulinum treatment of cranio-cervical dystonia, spasmodic dysphonia, other focal dystonias and hemifacial spasm. J Neurol Neurosurg Psychiatry 1990;53: 633-9.

15. Yoshida K, Iizuka T. Botulinum toxin treatment for upper airway collapse resulting from temporomandibular joint dislocation due to jaw-opening dystonia. Cranio 2006;24:217-22.

16. Yoshida K. Sensory trick splint as a multimodal therapy for oromandibular dystonia. J Prosthodont Res 2018 (in press)

17. Kaji R, Rothwell JC, Katayama M, et al. Tonic vibration reflex and muscle afferent block in writer's cramp. Ann Neurol 1995;38:155-62.

18. Yoshida K, Kaji R, Kohara N, et al. Movement-related cortical potentials before jaw excursions in patients with oromandibular dystonia. Mov Disord 2003;18:94-100.

19. Yoshida K, Iizuka T. Jaw-deviation dystonia evaluated by movement-related cortical potentials and treated with muscle afferent block. Cranio 2003;21:295300 .

20. Steeves TD, Day L, DykemanJ, et al. The prevalence of primary dystonia: a systematic review and meta-analysis. Mov Disord 2012;27:1789-96.

21. Wechsler LR, Tsao JW, Levine SR, et 
al. Teleneurology applications: report of the Telemedicine Work Group of the American Academy of Neurology. Neurology 2113;80:670-6.

22. Achey M, Aldred JL, Aljehani N, et al. The past, present, and future of telemedicine for Parkinson's disease.
Mov Disord 2014;29:871-83.

23. Agnihotri SP, Koralnik IJ. Training for a neurology career in a rare disease: the role of cyberconsults. Ann Neurol 2015;77:738-40.

24. Yoshida K. Computer-aided design/ computer-assisted manufacture-derived needle guide for injection of botulinum toxin into the lateral pterygoid muscle in patients with oromandibular dystonia. J Oral Facial Pain Headache 2018 (in press). 